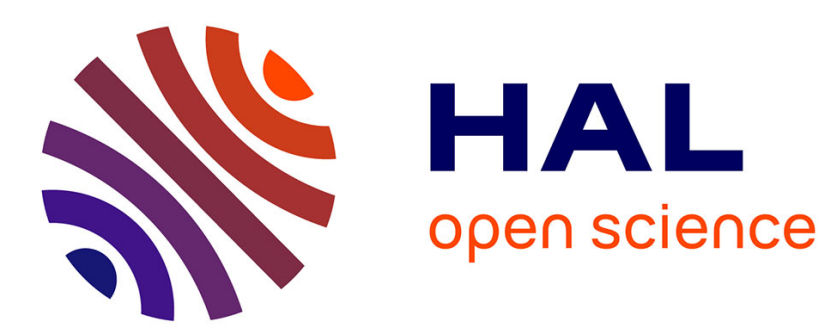

\title{
An alternative theoretical formula for hemoglobin oxygenation.
}

Denis Michel

\section{To cite this version:}

Denis Michel. An alternative theoretical formula for hemoglobin oxygenation.. European Biophysics Journal, 2008, 37 (6), pp.823-7. 10.1007/s00249-008-0283-2 . hal-00290678

\section{HAL Id: hal-00290678 \\ https://hal.science/hal-00290678}

Submitted on 22 Mar 2010

HAL is a multi-disciplinary open access archive for the deposit and dissemination of scientific research documents, whether they are published or not. The documents may come from teaching and research institutions in France or abroad, or from public or private research centers.
L'archive ouverte pluridisciplinaire HAL, est destinée au dépôt et à la diffusion de documents scientifiques de niveau recherche, publiés ou non, émanant des établissements d'enseignement et de recherche français ou étrangers, des laboratoires publics ou privés. 


\title{
An alternative theoretical formula for hemoglobin oxygenation
}

\section{Denis Michel}

Molecular and Cellular Interactions. Université de Rennes1

CNRS UMR6026 Hip IFR140. Campus de Beaulieu. Bat. 13. 35042 Rennes cedex. France.

\begin{abstract}
Classical models of homotropic allostery are based on the postulate that the binding sites are equivalent in their ability to interconvert between high and low affinity states, but compelling evidence exists that the subunits of human hemoglobin are not simultaneously available for oxygen equilibration, thus reducing the number of possible intermediate microstates. The incorporation of these results into the Adair scheme reveals an alternative mechanism for hemoglobin oxygenation, not based on affinity changes.
\end{abstract}

Key words Hemoglobin; subunits; allostery; cooperative binding; Hill plot; sequential equilibration mechanism

E. mail addresses: denis.michel@univ-rennes1.fr; denis.michel@live.fr 


\section{Introduction}

The sigmoidal shape of hemoglobin $(\mathrm{Hb})$ oxygenation inspired the intuitive idea that the binding of the first oxygen molecules increases the affinity for oxygen in the following binding steps (Bohr et al. 1904). Models of homotropic allostery based on this assumption yielded equations fitting experimental curves, when adjusting the values of theoretical constants. These models postulate that the initial fixation of the ligand leads to an increase in the affinity for the ligand of the remaining binding sites, in a concerted (Monod et al. 1965) or sequential (Koshland et al. 1966) manner. Vertebrate hemoglobin is made of two types of subunits with distinct kinetic and affinity properties. A series of convincing observations showed that the $\alpha$ and $\beta$ subunits of human $\mathrm{Hb}$ are differentially liganded at intermediate ligand concentration (Lindstrom and Ho 1972; Brzozowski et al. 1984; Simonneaux et al. 1988). Before discussing the biophysical significance of these results, their theoretical influence on saturation curves are examined. This analysis suggests that the organization of hemoglobin could also have been evolutionary optimized to generate cooperative effects without need for affinity changes.

\section{Theory}

The saturation ratio $Y$ of a macromolecule containing several binding sites for a ligand $\mathrm{L}$, is given by the Adair general equation (Adair 1925), whose microscopic formulation for a symmetric molecule can be written as in Eq. 1, where $n$ is the number of binding sites and $K_{\mathrm{j}}$ is intrinsinc binding constant of the $j^{\text {th }}$ binding step.

$$
Y=\frac{\sum_{i=1}^{n} i\left(\begin{array}{c}
n \\
i
\end{array}\right)\left(\prod_{j=1}^{i} K_{j}\right)[L]^{i}}{1+\sum_{i=1}^{n}\left(\begin{array}{c}
n \\
i
\end{array}\right)\left(\prod_{j=1}^{i} K_{j}\right)[L]^{i}}
$$

When the binding sites are identical and non-interacting, Eq. 1 dramatically reduces to the simple hyperbola $K[L] /(1+K[L])$. This reduction can be avoided through several ways: i) by a stepwise increase of the affinity for the ligand, according to Bohr's assumption, and also ii) by reduction of the number of possible intermediate states. This last possibility can be achieved, for example, when certain subunits of the multimer are prevented from ligand binding until 
others are liganded. Let us postulate that one group of components, called second (S) equilibrates with the ligand only when a group of components called first $(\mathrm{F})$ is liganded. Let be $K \mathrm{~s}$ and $K_{\mathrm{f}}$ the intrinsic association constants of the $\mathrm{S}$ and $\mathrm{F}$ components, respectively, and let be a multimer containing $2 n$ subunits, including $n$ equivalent $\mathrm{F}$ components and $n$ equivalent $\mathrm{S}$ components. To determine the shape of the equilibrium curve expected in this case, one can theorize the binding reaction with a modified Adair scheme as shown in Eq. 2. The $2 n$ successive binding steps are expressed with only two microscopic constants: $K_{\mathrm{f}}$ for the binding steps 1 to $n$, and $K$ s for the binding steps $(n+1)$ to $2 n$, associated with combinatorial factors reflecting the functional equivalence of the $n$ binding sites from each group. This hierarchical equilibration of two groups of $n$ binding sites leads to combinatorial terms different from those obtained for the global equilibration of $2 \mathrm{n}$ binding sites with the ligand (Eq. 1 where $n$ is replaced by $2 n$ ).

$$
Y=\frac{\sum_{i=1}^{n} i\left(\begin{array}{c}
n \\
i
\end{array}\right)\left(K_{f}[L]\right)^{i}+\left(K_{f}[L]\right)^{n} \sum_{i=1}^{n}(n+i)\left(\begin{array}{c}
n \\
i
\end{array}\right)\left(K_{s}[L]\right)^{i}}{2 n\left(1+\sum_{i=1}^{n}\left(\begin{array}{c}
n \\
i
\end{array}\right)\left(K_{f}[L]\right)^{i}+\left(K_{f}[L]\right)^{n} \sum_{i=1}^{n}\left(\begin{array}{c}
n \\
i
\end{array}\right)\left(K_{s}[L]\right)^{i}\right)}
$$

Let be $a=\frac{K_{\mathrm{S}}}{K_{\mathrm{f}}}$ and $x=K_{\mathrm{f}}[L]$

Eq. 2 becomes

$$
Y=\frac{\sum_{i=1}^{n} i\left(\begin{array}{c}
n \\
i
\end{array}\right) x^{i}+x^{n} \sum_{i=1}^{n}(n+i)\left(\begin{array}{c}
n \\
i
\end{array}\right)(a x)^{i}}{2 n\left(1+\sum_{i=1}^{n}\left(\begin{array}{c}
n \\
i
\end{array}\right) x^{i}+x^{n} \sum_{i=1}^{n}\left(\begin{array}{c}
n \\
i
\end{array}\right)(a x)^{i}\right)}
$$

Using the binomial identity and its derivative, Eq. 3 can be transformed into:

$$
Y=\frac{x(1+x)^{n-1}+x^{n}\left((1+2 a x)(1+a x)^{n-1}-1\right)}{2\left((1+x)^{n}+x^{n}\left((1+a x)^{n}-1\right)\right)}
$$

The system can be extended to multimers containing more than 2 groups of subunits. Eq. 5 corresponds to $p$ groups successively available for binding, each of them including $n$ subunits with the same intrinsic affinity constant $K_{\mathrm{i}}$. The ratio $K_{\mathrm{i}} / K_{\mathrm{f}}$ is written $a_{\mathrm{i}}$. 
$Y=\frac{\sum_{i=1}^{p}\left(\left(\prod_{j=1}^{i-1} a_{j}\right) x^{i-1}\right)^{n}\left(\left(i-1+i a_{i} x\right)\left(1+a_{i} x\right)^{n-1}-i+1\right)}{p\left(1+\sum_{i=1}^{p}\left(\left(\prod_{j=1}^{i-1} a_{j}\right) x^{i-1}\right)^{n}\left(\left(1+a_{i} x\right)^{n}-1\right)\right)}$

If every group contains only one member $(\mathrm{n}=1)$ with the same affinity for the ligand $\left(a_{\mathrm{i}}=1\right)$, Eq. 5 expectedly reduces to Eq. 6 which corresponds to the ordered binding scheme, a limit case with minimal number of microcanonical components and with a predicted Hill coefficient equal to $(p+2) / 3$ (Michel 2007).

$Y=\frac{\sum_{i=1}^{p} i x^{i}}{p \sum_{i=0}^{p} x^{i}}$

The Hill representation $\ln (Y /(1-Y))=\mathrm{f}\left(\ln \left(K_{\mathrm{f}}[L]\right)\right)$, crossing the abscissa at mid-saturation and converting an hyperbola into a straight line with unity slope, is currently used for improved visualization. The maximal slope of this curve, known as the Hill coefficient, is considered as an index of the degree of apparent cooperativity. The theoretical Hill curve equation in normal coordinates can be obtained from Eq. 4 when setting $X=\ln \left(K_{\mathrm{f}}[L]\right)$ and $H=\ln (Y /(1-Y))$ :

$H=\ln \frac{\mathrm{e}^{X}\left(1+\mathrm{e}^{X}\right)^{n-1}+\mathrm{e}^{n X}\left(\left(1+2 a \mathrm{e}^{X}\right)\left(1+a \mathrm{e}^{X}\right)^{n-1}-1\right)}{\left(2+\mathrm{e}^{X}\right)\left(1+\mathrm{e}^{X}\right)^{n-1}+\mathrm{e}^{n X}\left(\left(1+a \mathrm{e}^{X}\right)^{n-1}-1\right)}$

Figure 1 shows curves obtained using Eqs. 4 and 7 when $n=2$ (corresponding to a tetramer made of 2 types of protomers, like human $\mathrm{Hb}$ ). Saturation and Hill curves are sigmoidal for relatively low values of $a$ and for $a=1$ (protomers of equal affinity for the ligand) but increasing the value of a strongly increases the maximal slope of the sigmoid curve. The predicted Hill curves display the typical shape of experimental Hill plots, with asymptotes of unity slope on both sides. Increasing either $a$ or $n$ increases the maximal slope of the central part of the curve (Fig. 1b). The complete absence of cooperative effect, reflected by a linear Hill curve with a constant unity slope, is obtained when $a=0.25$ and $n=1$. 


\section{Comparison with experimental data}

To compare these theoretical curves to experimental data, the saturation curve of human $\mathrm{Hb}$ by the ligand phosphine $\mathrm{PMe}_{3}$ (Simonneaux et al. 1988) is selected since it has precisely been obtained in experimental conditions that allowed to conclude a preferential filling of the $\alpha$ subunit at low ligand concentration (Simonneaux et al. 1988). As shown in Fig. 2, the theoretical curve obtained from Eq. 4 remarkably fits the experimental points obtained by these authors, when using the following combination: $n=2, a=3$ and $K_{\mathrm{f}}=0.12 \mathrm{mM}^{-1}$. According to this scheme, binding curves can be predicted without arbitrary assignment of constant values but using virtually measurable parameters: $n$ and $a$. It would be interesting to compare the value $a=3$ with the real intrinsic constants of the $\alpha$ and $\beta$ subunits of $\mathrm{Hb}$, but unfortunately data from the literature are too conflicting to draw reliable conclusions about these equilibrium constants. Indeed, if determining $n$ is simple, the accurate assessment of individual binding constants is a notoriously difficult task. Equilibrium data from different laboratories, but also from different data sets, are often discordant. The procedure of dissociation of protomers can alter their properties and conversely, results obtained with holomultimers are affected by quaternary interactions and heretotropic factors. In addition, the values of equilibrium constants are sometimes deduced by assuming particular theoretical mechanisms, thus biasing the results, or calculated from the reaction rates, which might be misleading if association and dissociation rates are in fact generated by different $\mathrm{Hb}$ conformations.

The Hill plots which have long proved useful for evaluating the degree of cooperativity, can also be helpful for determining the affinity constants and the ratio $a$. If supposing that a saturation process is only governed by the present mechanism, the equations of the 2 asymptotes of the Hill plot are, at low ligand concentration: $\mathrm{A}_{1}(X)=X-\ln 2$ and at high ligand concentration: $\mathrm{A}_{2}(X)=X+\ln a+\ln 2$. The Hill curve crosses the abscissa at $X=-$ $(\ln 2) / 2$, and the normal distance $D$ between the two asymptotes $\mathrm{A}_{1}$ and $\mathrm{A}_{2}$ is $D=(\ln a+$ $\ln 4) / \sqrt{2}$, in accordance with the general formula (Michel 2007), in this particular system. 


\section{Plausibility of this mechanism for Human $\mathrm{Hb}$}

The key point of current models developed to explain the behaviour of $\mathrm{Hb}$ is the capacity of the individual binding constants to change depending on the saturation ratio. This postulate is no longer required in the present system, where every binding constant ( $K_{\mathrm{f}}$ or $K \mathrm{~s}$ ), remains indeed constant for a given binding site. This formula does not correspond to a model but to a simple equilibrium analysis assuming as an axioma, the sequential equilibration of the different types of protomers. Hence, the main question is to determine the validity of this axioma. Max Perutz discussed the possibility that only the $\alpha$ chains are filled at low oxygen concentration in the human $\mathrm{Hb}$, the $\beta$ chains being accessible to ligand only after both $\alpha$ chains are liganded (Perutz 1970). A valine residue (Val E11) next to the distal histidine could be involved in the stereochemical blockage of the $\beta$-heme pockets in the deoxy conformation (Bolton and Perutz 1970; Fermi et al. 1974) and, as explained by Perutz, the $\alpha$ subunits showed no such obstruction. Accordingly, crystallization of partially liganded tetramers revealed intermediate states, in which the $\alpha$ chains are oxygenated and the $\beta$ subunits are oxygen-free (Brzozowski et al. 1984). Chemical shift NMR analyses, which are valuable tools to identify intermediate states in stable equilibrium conditions, also demonstrated the same dissymmetrical filling of $\mathrm{Hb}$ subunits by oxygen (Lindstrom and Ho 1972) and by the bulky ligand phosphine (Simonneaux et al. 1988). This situation is likely to not have a thermodynamic origin since, even if their values fluctuate between the reports, no drastic differences between the equilibrium constants of the $\alpha$ and $\beta$ chains have generally been reported. Interestingly, studies on subunits isolated from human $\mathrm{Hb} \mathrm{A}$, showed that isolated $\beta$ subunits have clearly higher affinity for oxygen than isolated $\alpha$ subunits (Riggs and Gibson 1973). Hence, the two types of binding sites seem to not compete in the same equilibrium in the lower range of ligand concentration. Together, these observations suggest, as anticipated by Perutz 1970, a molecular organisation in which the $\beta$ sites become receptive to oxygen only after clicking the tetramer to an oxy conformation following the saturation of the $\alpha$ chains, so that the ligand binds first to the $\alpha$ sites and dissociates first from the $\beta$ sites (Simonneaux et al. 1988). This system could have been evolutionary selected for its ability to make possible, an otherwise thermodynamically unacceptable situation in which the filling of the low affinity sites is obtained at lower ligand concentration than that of the high affinity ones. 


\section{Discussion}

It is admitted that the sigmoidal shape of oxygenation curves results from tridimensional rearrangements in $\mathrm{Hb}$ called allostery, from the Greek roots allos: other and stereos: solid, shape. Many changes of the $\mathrm{Hb}$ quaternary structure have indeed been associated with vertebrate $\mathrm{Hb}$ oxygenation such as, among others, the well-established movement of the iron atom in the porphyrin plane, considered as a triggering event, or the hindrance of the ligand site of the $\beta$ subunits in deoxy-Hb. The different models and interpretations then discern in this set of molecular changes, the causal events more directly involved in the genesis of sigmoid equilibrium curves. The hypothesis presented here proposes that beside mechanisms related to affinity changes, certain structural interactions occurring in human $\mathrm{Hb}$ could be involved in dictating the binding sequence through the rearrangement of subunits. The equations resulting from this sequential equilibration hypothesis have interesting features when compared to those generated by the classical models, such as symmetrical Hill curves and no need for large differences between binding constants. In the example presented here, a three times higher affinity of the second component is sufficient to fit the experimental data (Fig. 2), which is relatively low, as compared to the 70-fold increase of the affinity for oxygen during the $\mathrm{T}$ to $\mathrm{R}$ conversion postulated by Jacques Monod for an optimal adjustment of the MWC mathematical equation to an experimental equilibrium curve $(L=9,054$ and $c=0.014$, Monod et al. 1965). The differential behaviour of the $\alpha$ and $\beta$ subunits, so far intriguing and neglected in the literature and for which the classical models of allostery are quite ensuited, provides, in fact, a solution to generate sigmoid saturation curves without changing the binding constants of individual subunits and a possible explanation for the presence of different types of subunits in vertebrate $\mathrm{Hb}$. This organization could be optimized to generate pronounced sigmoid equilibrium curves using minimal requirements: i) different types of subunits successively available for binding and ii) only two subunits of each type since a limited value of $n$ is sufficient in this system, contrary to the basic ordered scheme, which is more appropriate to giant respiratory proteins where identical subunits are polymerised (Michel 2007). The present study is, however, a simplification of the real situation since it does not take into account many other parameters such as heterotropic factors or the capacity of the $\mathrm{Hb}$ tetramer to dissociate into dimers, but it could describe an additional facet of the cooperative oxygenation of vertebrate $\mathrm{Hb}$. 


\section{References}

Adair GS (1925) The hemoglobin system. VI. The oxygen dissociation curve of hemoglobin. J Biol Chem 63: 529-545.

Bohr C, Hasselbach KA, Krogh A (1904) Skand Arch Physiol 16: 401-412.

Bolton W, Perutz MF (1970) Three dimensional fourier synthesis of horse deoxyhaemoglobin at 2.8 Angstrom units resolution. Nature 228: 551-552.

Brzozowski A, Derewenda Z, Dodson E, Dodson G, Grabowski M., Liddington R., Skarzyn'ski T, Vallely D. (1984) Bonding of molecular oxygen to T state human haemoglobin. Nature 307: 74-76.

Fermi G, Perutz MF, Shaanan B, Fourme, R (1974) The crystal structure of human deoxyhaemoglobin at $1.74 \AA$ resolution. J Mol Biol 175: 159-174.

Koshland DEJ, Nemethy G, Filmer D (1966) Comparison of experimental binding data and theoretical models in proteins containing subunits. Biochemistry 5: 365-385.

Lindstrom TR, Ho, C (1972) Functional nonequivalence of $\alpha$ and $\beta$ hemes in human adult hemoglobin. Proc Natl Acad Sci USA 69: 1707-1710.

Michel D (2007) Cooperative equilibrium curves generated by ordered ligand binding to multi-site molecules. Biophys Chem 129: 284-288.

Monod J, Wyman J, Changeux J-P (1965) On the nature of allosteric transitions: a plausible model. J Mol Biol 12: 88-118.

Perutz MF (1970) Stereochemistry of cooperative effects in haemoglobin. Nature 228: 726739.

Riggs AF, Gibson QH (1973). Oxygen equilibrium and kinetics of isolated subunits from hemoglobin Kansas. Proc Natl Acad Sci USA 70: 1718-1720.

Simonneaux G, Bondon A, Brunel C, and Sodano P (1988) Direct observation of intermediate ligation states of hemoglobin. J Am Chem Soc 110: 7637-7640. 


\section{Figures}
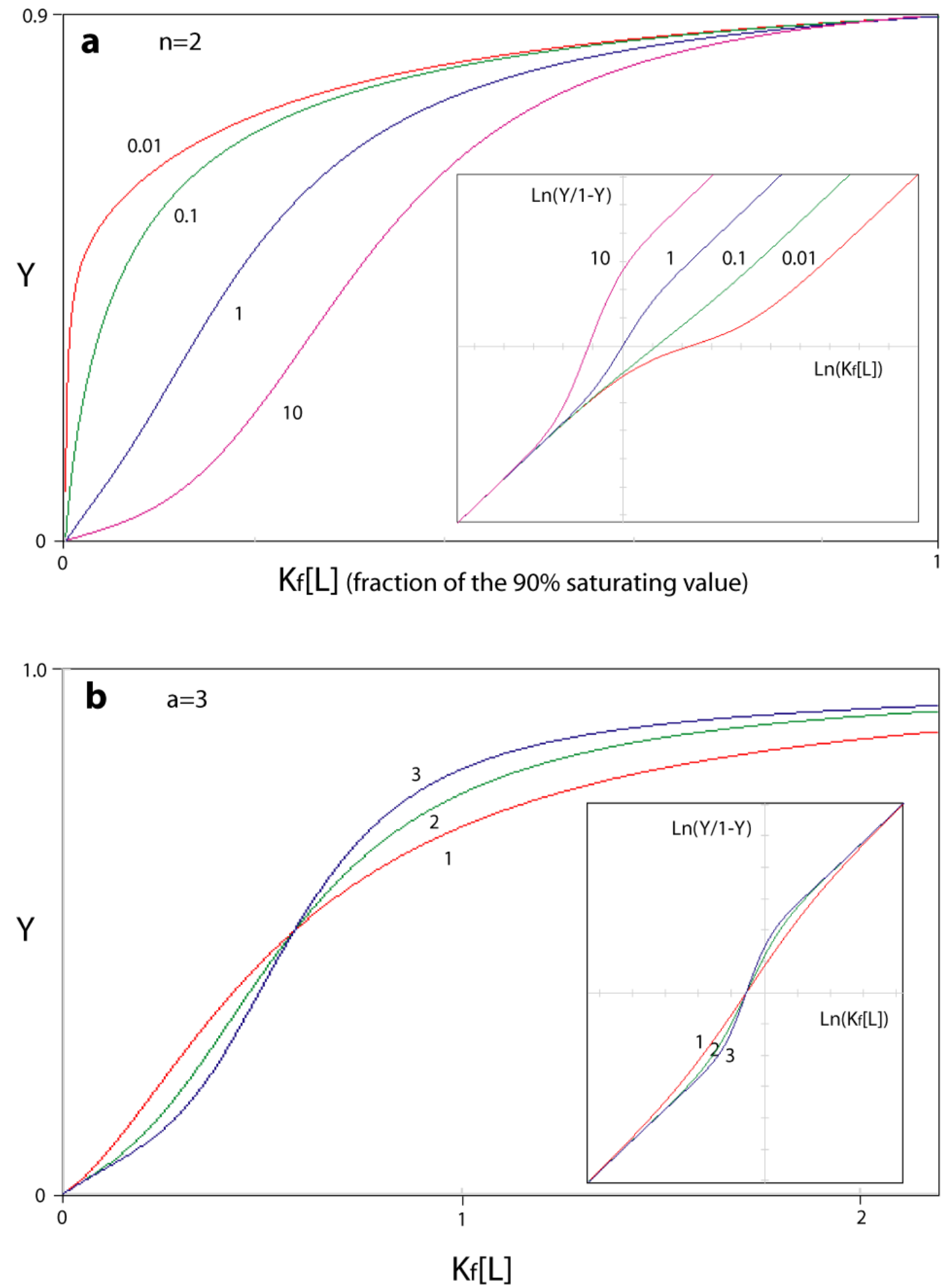

Fig. 1 Visualization of the curve shapes obtained from Eq. 4 with varying values of $n$ and $a$. (a) $n=2$ and varying a (red $a=0.01$, green $a=0.1$, blue $a=1$, purple $a=10$ ). (b) $a=3$ and varying $n$ (red $n=1$, green $n=2$, blue $n=3$ ). The corresponding Hill plots are shown in the small inserted panels 


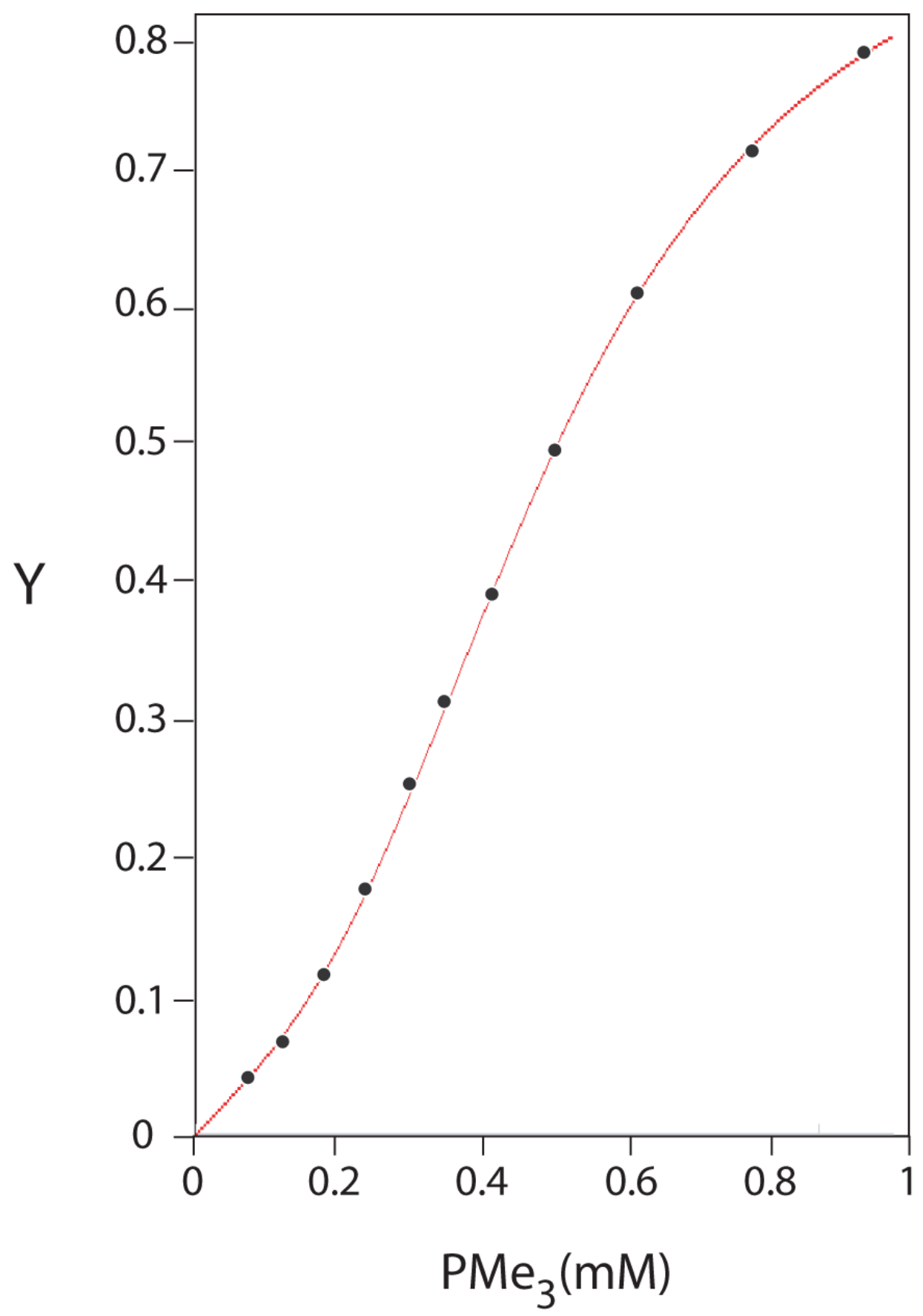

Fig. 2 Saturation of $\mathrm{Hb}$ with the phosphine $\mathrm{PMe}$. Results (black points) obtained from (Simonneaux et al. 1988). Solid line drawn to Eq. 4 using the values $n=2, a=3$ and $K_{\mathrm{f}}=$ $0.12 / \mathrm{mM}$ 